\title{
Relationship between the density of tatami stores and COVID-19 morbidity and mortality in Japan
}

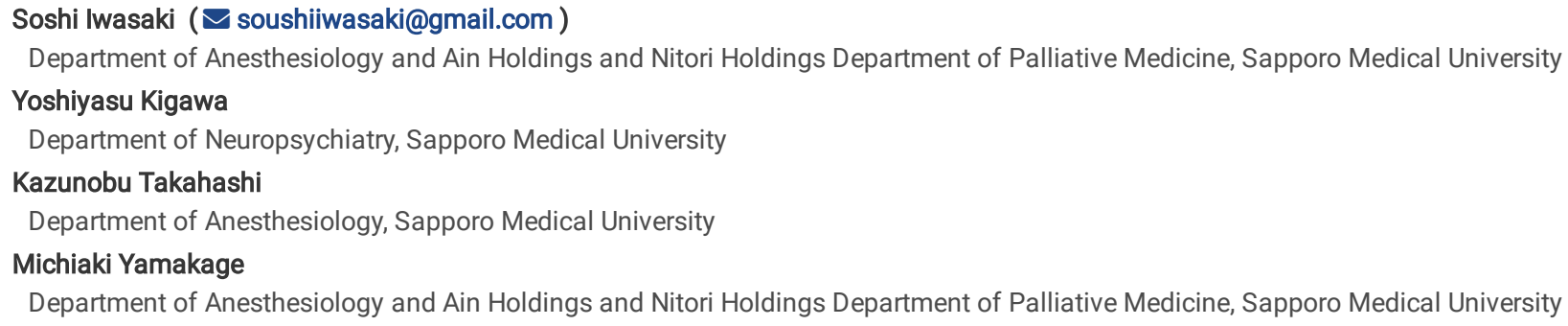

\section{Short Report}

Keywords: COVID-19, shoe-related transmission, tatami stores, mortality, morbidity, population density, Japanese-style room

Posted Date: November 24th, 2020

DOI: https://doi.org/10.21203/rs.3.rs-112449/v1

License: (9) (1) This work is licensed under a Creative Commons Attribution 4.0 International License. Read Full License 


\section{Abstract}

Although the primary mechanism for the spread of coronavirus disease 2019 (COVID-19) infection is through respiratory droplets (1), the virus that causes COVID-19 can survive on smooth surfaces (e.g., stainless steel, plastic, glass) for days at low temperatures (2). Surface transmission represents a possible route of infection, as unwashed hands that touch a contaminated surface can transfer the virus to mucous membranes (e.g., eyes, nose, mouth) (3). However, it is difficult to observe disease transmission directly. Therefore, the Japanese Cluster Countermeasures team has identified the "Three Cs"-Closed spaces with poor ventilation, Crowded places, and Close-contact settings-as factors to avoid to prevent COVID-19 clusters. Identifying other environmental factors that increase or decrease the number of infections would be useful to prevent the spread of COVID-19. Therefore, this study aimed to develop a shoe-related transmission model and enhance COVID-19 transmission route precautions by investigating the associations between the prevalence of COVID-19 and both population density and the distribution of tatami stores in each prefecture in Japan. As a result, a significant negative relationship was observed between the number of tatami stores per 100,000 population and the number of COVID-19 cases and deaths.

\section{Introduction}

Tatami is a type of straw mat used for flooring in Japanese-style rooms. Since Japanese people always take off their shoes before entering a Japanese-style room, the density of tatami stores in a locale might be a proxy for the cultural practice of shoe removal in Japan. Therefore, we hypothesized that the number of tatami stores in each prefecture, adjusted by population, would be related to the number of cases of, and deaths associated with, coronavirus disease 2019 (COVID-19) in Japan. We previously reported that there were significantly fewer deaths associated with CoVID-19 relative to population size in countries that follow the cultural practice of removing shoes when going indoors compared with those that do not (4).

Globally, regional differences are seen in the morbidity and mortality rates for COVID-19 infection. In general, patients with COVID-19 tend to reside in densely populated cities (5). In Japan, as of mid-2020, the number of infected people per capita is the highest in Tokyo Prefecture, followed by Hokkaido, Osaka, Fukuoka, Aichi, and Okinawa Prefectures (6).

However, the number of infections is high even in places with less dense populations, such as Hokkaido, the least populous of Japan's 47 prefectures. One of the reasons for the high number of reported COVID-19 cases in Hokkaido is that it is located in the northernmost part of Japan, where the temperature is cold. However, the Tohoku region, which is just south of Hokkaido, has reported far fewer cases of COVID-19 infections, whereas Okinawa, which is located in the southernmost, tropical part of Japan, has reported many cases. Despite these regional differences, there is something common to areas across Japan: tatami flooring.

The tatami culture in Japan, including the practice of always removing one's shoes before stepping onto a tatami mat, persists to the present day. Given this background, this study aimed to develop a shoe-related transmission model and enhance COVID-19 transmission-route precautions by investigating the association between the prevalence of COVID-19 and both population density and the distribution of tatami stores in each prefecture in Japan.

\section{Materials And Methods}

To test the validity of our hypothesis, we compared the number of COVID-19 cases and deaths with the number of tatami dealers in all 47 prefectures in Japan and the population density in each prefecture. Data regarding prefectural populations and population density were obtained from the 2015 Census (7). The number of COVID-19 cases were obtained from NHK's dedicated COVID-19 website (8) and the number of deaths from Google's special site (9) (Data were obtained by Google from authoritative sources such as Wikipedia, national health ministries, and The New York Times).

There are two large tatami associations in Japan: the Japan Tatami Promotion Association (10) and Zennihon Tatamijigyo Kyodokumiai (11). Almost all tatami stores register with one or both associations. The tatami stores in each prefecture were counted from the homepages of these associations. The obtained information was collated, and duplicate tatami stores were excluded. A scatterplot of the number of tatami stores per 100,000 population and COVID-19 cases and deaths was created for the statistical analysis.

To evaluate the relationship between the numbers of tatami stores and COVID-19 cases and deaths, rate ratios were calculated using "the number of COVID-19 cases and deaths" as the dependent variable and "the number of tatami stores per 100,000 people" as the independent variable, with the confidence interval (Cl) set at $95 \%$. This formulation of the independent variable corrected for differences in regional populations. Additionally, a Poisson regression model using a logarithm as a link function was analyzed.

Similarly, to evaluate the relationship between "population density" and "the number of COVID-19 cases and deaths," rate ratios were calculated, with the "number of COVID-19 cases" and "number of COVID-19 deaths" set as the dependent variables and "population density" set as the independent variable. Similarly, 95\% Cls were used. A Poisson regression model was also analyzed. SPSS 22.0 (IBM Japan, Ltd., Tokyo, Japan) was used for the statistical analysis.

\section{Results}

A significant positive relationship was found between population density and the numbers of COVID-19 cases ( $p<0.001$; Fig. 1) and deaths ( $p<0.001$; not shown in the figure). The numbers of COVID-19 cases and deaths increased by 1.838 (95\% Cl: $1.828-1.848$ ) and 1.832 times (95\% Cl: $1.788-1.877$ ) respectively for every 1,000-person increase in population density. Hokkaido Prefecture was located off the curve of the scatterplot, at a single point.

A significant negative relationship was found between "the number of tatami stores per 100,000 population" and "the number of COVID-19 cases" ( $p<0.001$; Fig. 2) and "the number of COVID-19 deaths" ( $p<0.001$; not shown in the figure)." The number of COVID-19 cases and deaths increased by 0.547 (95\% Cl: 
$0.542-0.553)$ and 0.498 (95\% Cl: $0.475-0.521)$ times with every increase in the number of tatami stores per 100,000 population. In this case, Hokkaido Prefecture was located on the curve of the scatterplot.

\section{Discussion}

In terms of population density, Hokkaido Prefecture reported many COVID-19-related cases and deaths that deviated from the scatterplot. While high infection rates are reasonably expected in densely populated prefectures, Hokkaido Prefecture has a low population density; yet, the number of infections is relatively high. As such, it is necessary to build a simple model of COVID-19 infection patterns in Japan, including in outlier prefectures such as Hokkaido, that accounts for different regional development trajectories.

The development of Hokkaido began in earnest during the Meiji era, a period marked by the mass adoption of Westernization in Japan. Westernization had a substantial impact on the use of tatami in modern Japan, where tatami dealers have come under strong consumer selection pressure because of the spread of Western culture (12). Browsing each tatami store's website revealed that many established tatami stores, some of which have been in business for more than 100 years, continue to thrive in rural areas, with the exception being Hokkaido Prefecture. Hokkaido Prefecture has been Westernized since its development, resulting in fewer tatami stores. Lower numbers of tatami stores may be one reason why COVID-19 cases and deaths are high in Hokkaido. By contrast, the tatami industry in Taiwan, which is also facing selection pressure, continues to thrive (13). Today, Taiwan has achieved exceptional success in minimizing COVID-19 infections in the country.

While it is difficult to reduce population density, taking off one's shoes is well within an individual's control. To enter a Japanese-style tatami room, everyone must take off their shoes. If such rooms are kept clean, this cultural practice protects the family from visitors wearing shoes that may carry infected droplets.

Tatami rooms could be a factor that mitigates the spread of COVID-19 infection, but substantial time and resources are needed to create new Japanese-style rooms. Nonetheless, it is easy to take measures such as removing shoes worn in a crowded place before going indoors; therefore, removing one's shoes may be a viable option for preventing the spread of COVID-19 infection.

\section{Conclusion}

In this study, the number of tatami stores in each prefecture, adjusted by population, was negatively associated with COVID-19 morbidity and mortality, thereby providing a good model to explain the transmission of COVID-19 in Japan, including in cases such as Hokkaido Prefecture, which has a unique developmental history.

Regarding droplet transmission, the virus that causes COVID-19 can be found in the respiratory droplets of infected persons, which often fall to the ground. The National Institute of Infectious Diseases reported that on the Diamond Princess cruise ship, samples were collected from a total of 601 locations, and COVID-19 RNA was detected in 58. A sweep of patient's rooms returned 57 specimens from 21 rooms. The frequency of detection of the virus that causes COVID-19 varied in each room: 13 bathroom floors (39\%), 11 pillows (34\%), 8 telephones (24\%), 8 desks (24\%), and 7 TV remotes (21\%). The maximum number of days between guests leaving their room and the collection of RNA detection samples was 17 days (14).

As described in this paper, research on COVID-19 transmission suggests that it is dangerous to bring shoes from documented sites of COVID-19 cases into residential spaces. Therefore, an "Avoid the 'Three Cs'” campaign has been proposed to prevent the spread of infectious diseases such as COVID-19 (15). In addition to avoiding the "Three Cs" of Closed spaces with poor ventilation, Crowded places, and Close-contact settings, we propose "Care of shoes" as a fourth "C" to help prevent the spread of COVID-19 infection.

\section{Declarations}

Competing interests: The authors declare no competing interests.

\section{References}

1) Jayaweera M, Perera H, Gunawardana B, Manatungea J. Transmission of COVID-19 virus by droplets and aerosols: A critical review on the unresolved dichotomy. Environ Res. 2020; 188: 109819. doi:10.1016/j.envres.2020.109819

2) Doremalen N, Bushmaker T, Morris D, et al. Aerosol and surface stability of SARS-CoV-2 as compared with SARS-CoV-1. N Engl J Med. 2020; 382: 15641567. doi:10.1056/NEJMc2004973

3) Cevik M, Kuppalli K, Kindrachuk J, Peiris M. Virology, transmission, and pathogenesis of SARS-CoV-2. BMJ 2020; 371: m3862. doi:10.1136/bmj.m3862

4) Iwasaki S, Takahashi K, Yamakage M. The cultural practice of removing shoes indoors is relevant to the mortality rate of the SARS-CoV-2 pandemic. Res Square. doi:10.21203/rs.3.rs-32956/v1

5) Rocklöv J, Sjödin H. High population densities catalyze the spread of COVID-19. J Travel Med. 2020; 27(3): taaa038. doi:10.1093/jtm/taaa038

6) https://web.sapmed.ac.jp/canmol/coronavirus/japan.html (Retrieved 30 June 2020)

7) http://www.stat.go.jp/data/kokusei/2015/index.html (Retrieved 30 June 2020) 
8) https://www3.nhk.or.jp/news/special/coronavirus/data/ (According to the latest update on 30 June 2020)

9) https://www.google.com/search?

q=\%E3\%82\%B3\%E3\%83\%AD\%E3\%83\%8A+\%E8\%87\%B4\%E6\%AD\%BB\%E7\%8E\%87\&oq=\%E3\%82\%B3\%E3\%83\%AD\%E3\%83\%8A\%E3\%80\%80\%E8\%87\%B4\%E6\%

8 (According to the latest update on June 27, 2020, 20:00 GMT)

10) http://www.tatami.in/ (According to the latest update on June 27, 2020, 20:00 GMT)

11) http://www.tatami.or.jp/ (According to the latest update on June 27, 2020)

12) Tsai-Yun L. A survey on the status and legacy of Japanese tatami industry: An interview with the craftsmen.

https://www.jstage.jst.go.jp/article/jssd/62/0/62_223/_pdf/-char/en

13) Lo T-Y. Formation and historical development of tatami in Taiwan. Bulletin of JSSD. 2013. https://www.jstage.jst.go.jp/article/jssdj/60/3/60_3_29/_pdf

14) https://www.cdc.gov/coronavirus/2019-ncov/prevent-getting-sick/cloth-face-cover-guidance.html

15) Centers for Disease Control and Prevention. Considerations for wearing masks. https://www.niid.go.jp/niid/ja/diseases/ka/corona-virus/2019-ncov/2484idsc/9597-covid19-19.html

\section{Figures}

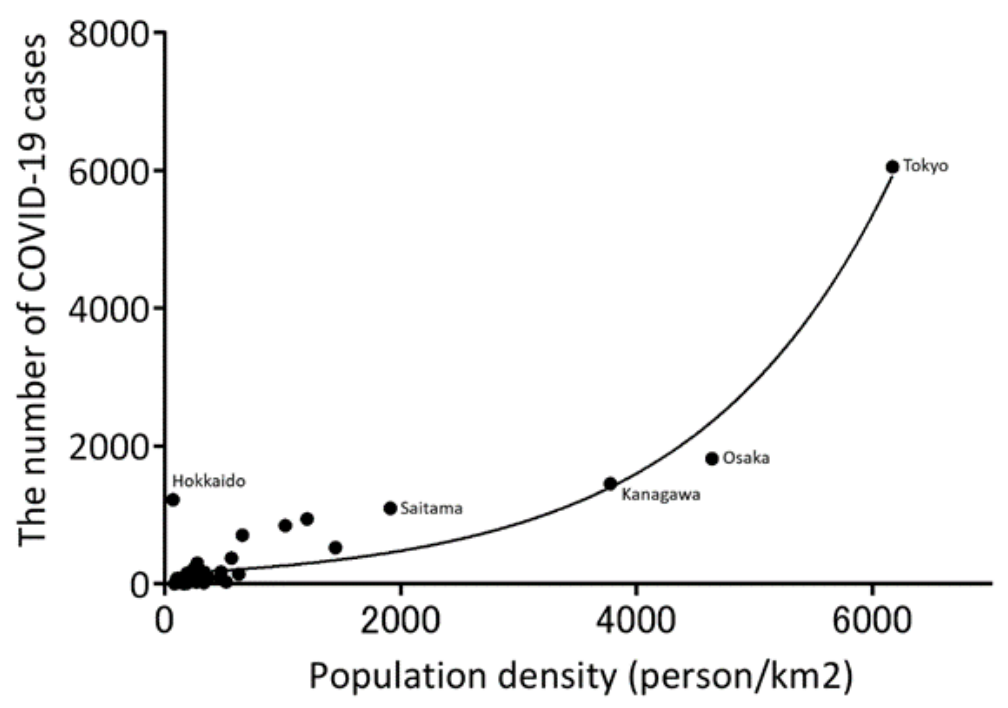

Figure 1

Relationship between population density and the numbers of COVID-19 cases and deaths.

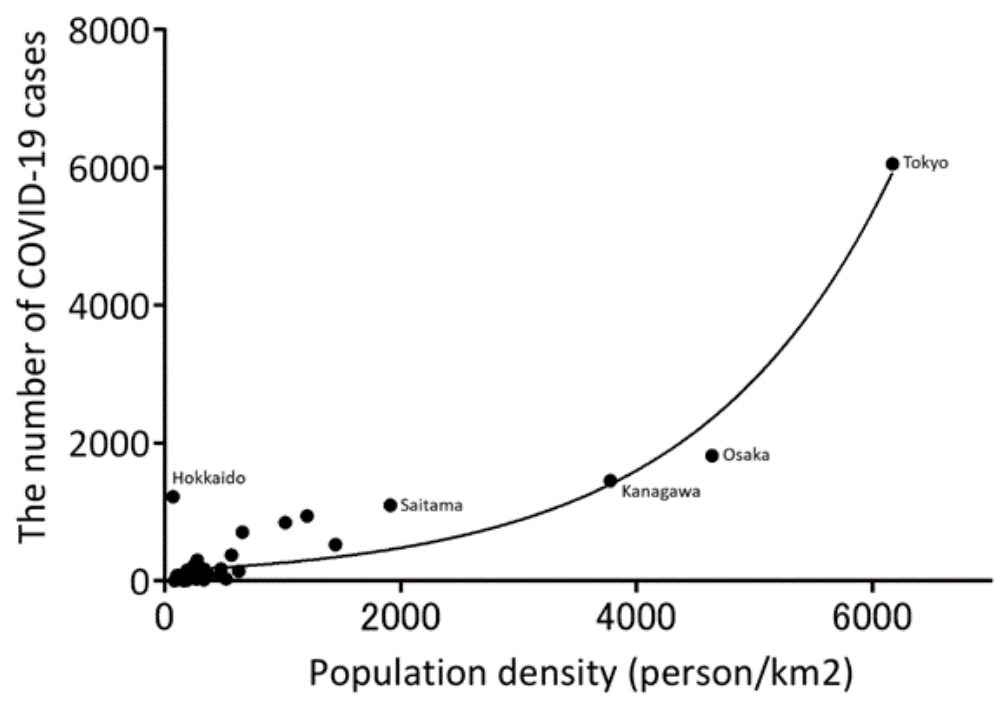

Figure 1

Page 4/5 
Relationship between population density and the numbers of COVID-19 cases and deaths.

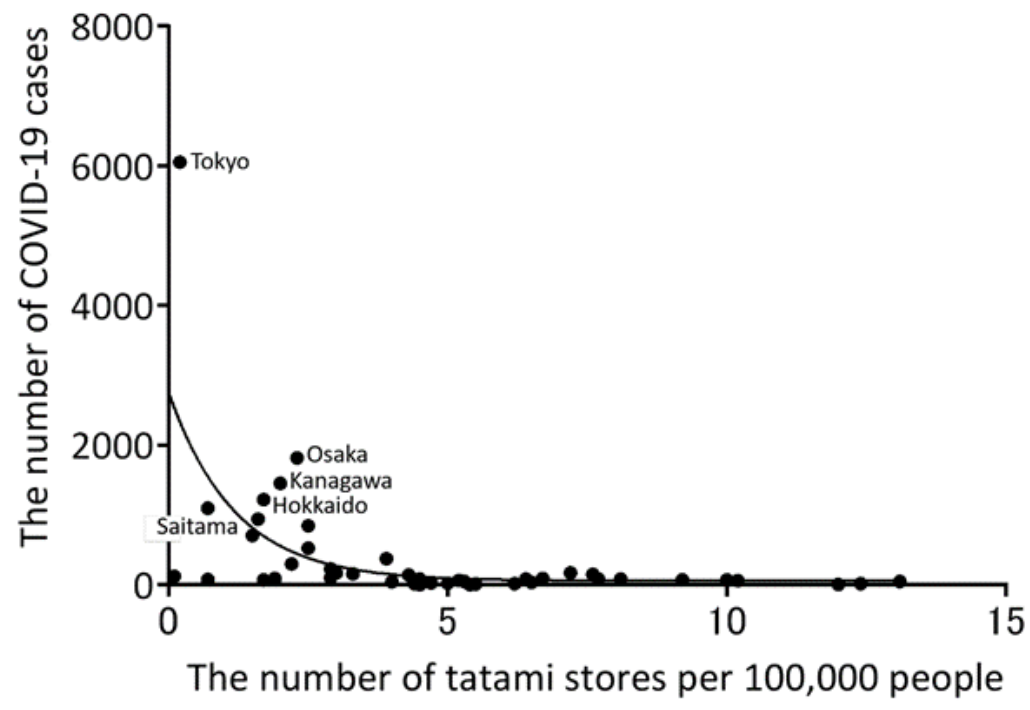

Figure 2

Relationship between the number of tatami stores per 100,000 people and the number of COVID-19 cases in each prefecture

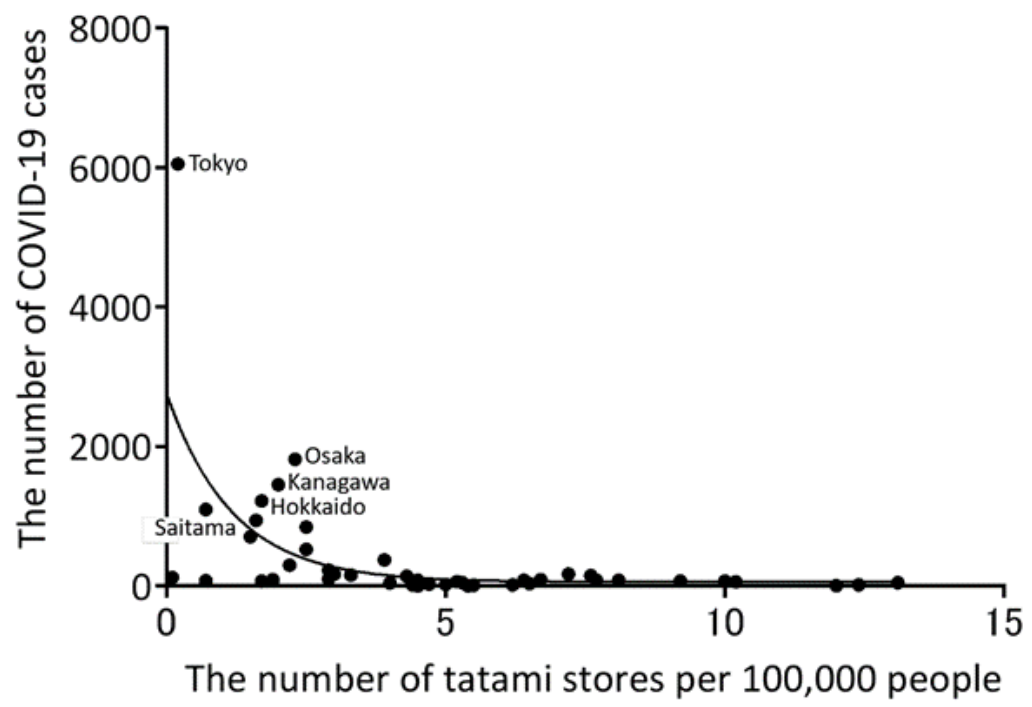

Figure 2

Relationship between the number of tatami stores per 100,000 people and the number of COVID-19 cases in each prefecture 